\title{
113. Cerebellar Projections to the Thalamic Nuclei in Monkeys ${ }^{\dagger)}$
}

\author{
By Toshio Kusama,*) Masako Mabuchi,*) and Teiko Sumino**) \\ (Comm. by Teizo OgawA, M. J. A., May 12, 1971)
}

Using the Nauta-Gygax method, Mehler et al.5) pointed out the Pf (pars dorso-lateralis), MD (paralaminar zone), small celled component of the Cl, VPLo, VLo, VA, area $\mathrm{X}$ and VAmc as the terminal nuclei of cerebellar fibers. This study offers some additional informations on this subject.

In five crab-eating monkeys (Macaca irus) total destruction of the superior cerebellar peduncle by electrical coagulation was attempted at the entrance of this peduncle to the brain stem (Fig. 1, C-D). It was successful in two monkeys (monkeys nos. 50 and 58). In these specimens lesions involved also the cerebellar and vestibular nuclei in different locations. They, however, were confined to one side, although they extended medially to include the rostral part of the medial cerebellar nuclei. In monkey no. 58 the involvement of the vestibular nuclei in lesion was so small that only the dorsomedial part of the superior nucleus and the dorsal end of the rostral part of the medial nucleus were destroyed. Lesion in monkey no. 50 was bigger and included the dorsal parts of the inferior, medial and lateral nuclei and the entire of the superior nucleus. Thus in the brain stem of these two monkeys only parts of the vestibular nuclei (Fig. 1, B-C) and surrounding area of the superior cerebellar peduncle (Fig. 1, D) were included in lesions.

For impregnation of degenerating fibers the Nauta-Gygax method $^{6)}$ was employed on every tenth of serial, frontal, frozen sections.

In monkey no. 58, furthermore, the adjoining sections were stained with the Fink-Heimer method. ${ }^{1)}$ In our specimens the Nauta-Gygax method resulted in good staining. In monkey no. 58 sections stained with the Nauta-Gygax and Fink-Heimer methods showed no significant difference in distribution and amount of degenerating fibers in the thalamic nuclei. Generally speaking, however, the Fink-Heimer method seemed to be more stable in staining

\footnotetext{
t) Conclusion of this study was cited in our atlas. ${ }^{3)}$

*) Department of Neuroanatomy, Institute of Brain Research, School of Medicine, University of Tokyo.

**) Department of Neurology, School of Medicine, Juntendo University.
} 

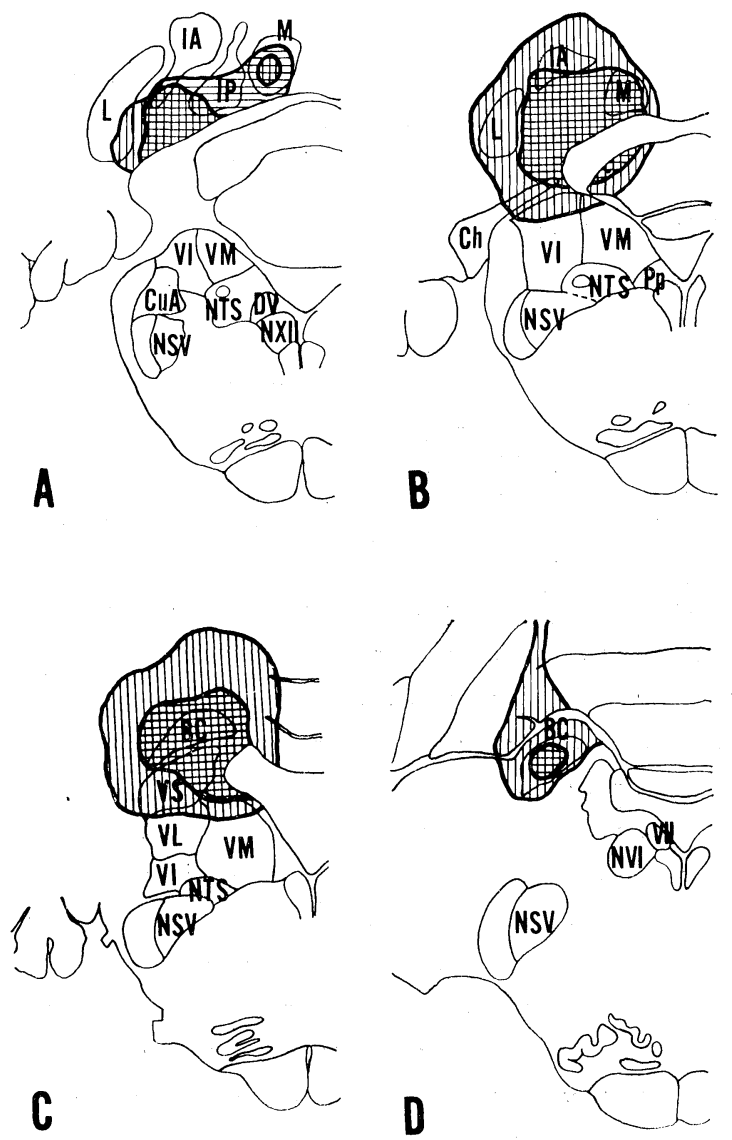

Fig. 1, A-D. Schematic representation of lesions in monkeys nos. 50 and 58. The former is hatched by vertical lines and the latter by horizontal lines. Abbreviations: BC, pedunculus cerebellaris superior; $\mathrm{Ch}$, nuclei cochleares; $\mathrm{CuA}$, nucleus cuneatus accessorius; DV, nucleus dorsalis $n$. vagi; IA, nucleus interpositus anterior cerebelli; IP, nucleus interpositus posterior cerebelli; L, nucleus lateralis cerebelli; $\mathrm{M}$, nucleus medialis cerebelli; NSV, nucleus tractus spinalis $n$. trigemini; NTS, nucleus tractus solitarii; NVI, nucleus $n$. abducentis; NXII, nucleus $n$. hypoglossi; Pp, nucleus prepositus hypoglossi; VI, nucleus vestibularis inferior; VL, nucleus vestibularis lateralis; VM, nucleus vestibularis medialis; VS, nucleus vestibularis superior; VII, radix $n$. facialis.

monkey brain sections. However, in sections stained with this procedure drawing of nuclear configurations by dark field microscopical observations was more difficult.

For mapping of degenerating fibers Olszewski's classification and abbreviations of the thalamic nuclei ${ }^{8)}$ were principally followed. 
Findings in monkeys nos. 50 and 58 with total destruction of the superior cerebellar peduncle were essentially the same and other monkeys with its partial destruction also did not demonstrate the contradictory findings.

On the ipsilateral side to lesions degenerating fibers caused by electrode tracks were found in the cerebral white matter, internal capsule and thalamic nuclei. As their differentiation from fibers of cerebellar origin was not simple, this paper will deal only with findings on the contralateral side.

A considerable amount of preterminal degenerating fibers were observed in the caudal part of the MD (paralaminar zone) and in the $\mathrm{Cl}$ (Fig. 2, A-D). In the Pcn only a slight number of degenerating fibers were found (Fig. 2, E). Most of many degenerating fibers in the CnMd-Pf complex were considered as passing fibers by their running pattern.

Degenerating fibers in the VPI, VPLc, VPM and VLm showed no definite pattern of terminations in these nuclei.

According to our cyto- and myeloarchitectural findings the extent of Olszewski's $\mathrm{VPLo}^{8)}$ seemed to be slightly wider in the caudal direction. The border between the VPLo and VPLc was considered to be located close to the rostral-most level of the nucleus centrum medianum. $^{3}$ ) Thus the VPLo at the +6.3 and probably +6.9 levels in Olszewski's atlas ${ }^{8)}$ appeared to belong to the VPLc. In our specimens preterminal degenerating fibers were distributed as dense networks throughout almost entire extent of the VPLo (Fig. 2, D and F). This finding was one of the reasons, by which the VPLo was designated as the nucleus ventralis intermedius (VI) in our atlas.3)

The VLc, especially its ventral part, showed dense preterminal degenerating fibers (Fig. 2, B-D). They, however, prominently diminished in number in the rostral end of the nucleus (Fig. 2, E). In the VLps a moderate amount of preterminal degenerating fibers were demonstrated. They increased in number in the rostral end of the nucleus (Fig. 2, A-B).

According to our cytoarchitectural study the VLo was not uniform, but consisted of two components: the first component was conspicuous, island-like clusters of medium-sized, darker cells and the second was slightly lighter cells of medium size which were disseminated among these clusters. Preterminal degenerating fibers observed in the VLo were small in number and distributed mostly among the clusters of cells. As shown in Fig. 2, E, most of them were observed in the region near to the lateral border of the VLo that belonged to the second component. Projections of precentral cortical area made preterminal degenerating fibers rather in the 


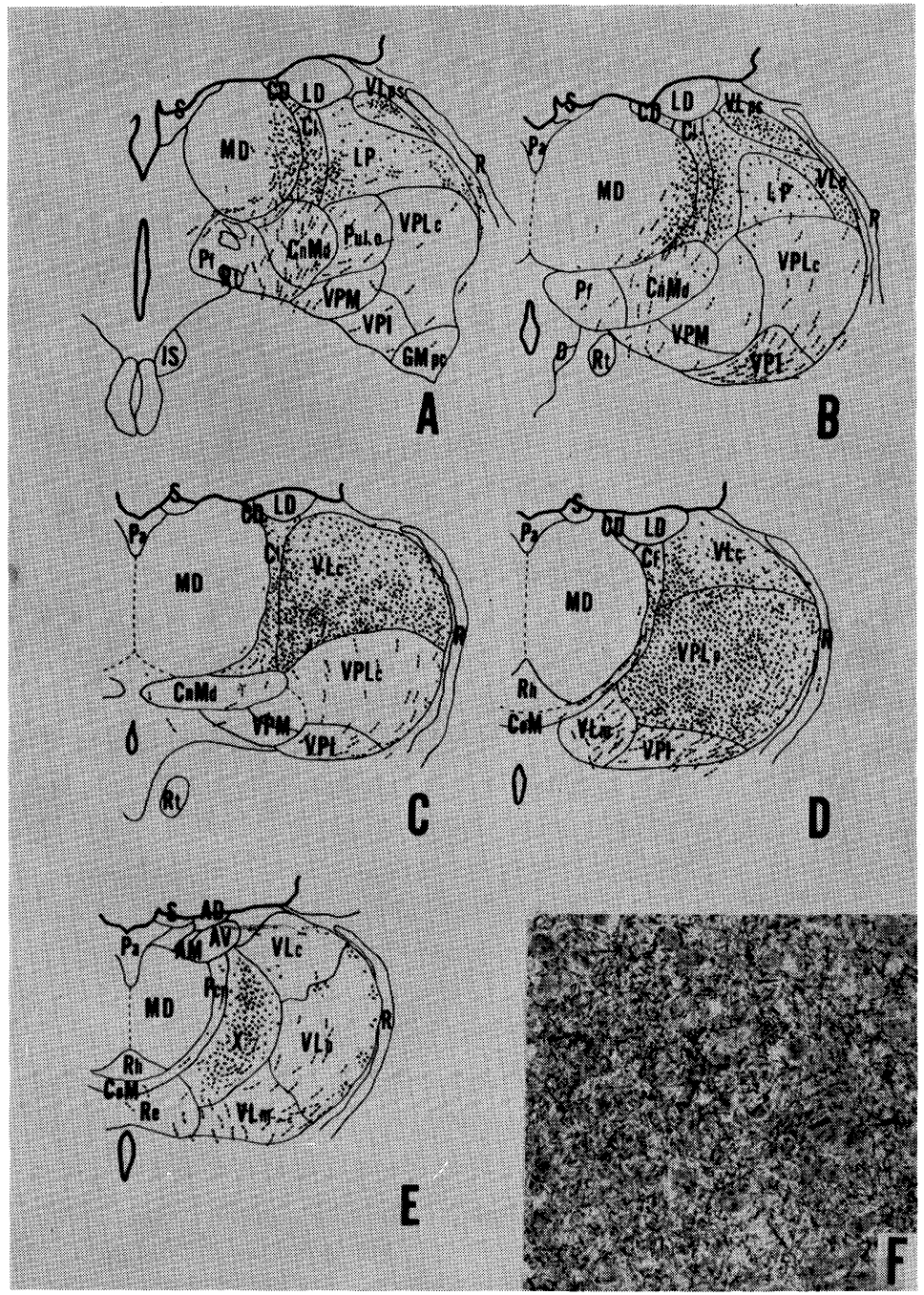

Fig. 2, A-E. Schematic representation of degenerating fibers in the contralateral thalamic nuclei following total destruction of the superior cerebellar peduncle in monkey no. 58. F, Photomicrograph shows degenerating fibers in the VI (VPLo) in sections stained with the NautaGygax method from the same monkey brain. $\times 100$ Abbreviations: $\mathrm{AD}$, nucleus anterior dorsalis; $\mathrm{AM}$, nucleus anterior medialis; $\mathrm{AV}$, nucleus anterior ventralis; CD, nucleus centralis dorsalis; CeM, nucleus centralis medialis; $\mathrm{Cl}$, nucleus centralis lateralis; $\mathrm{CnMd}$, nucleus centrum medianum; GMpc, nucleus geniculatus medialis, pars parvocellularis ; LD, nucleus lateralis dorsalis ; LP, nucleus lateralis posterior ; MD, nucleus medialis dorsalis; Pa, nucleus paraventricularis; Pcn, nucleus paracentralis; Pf, nucleus parafascicularis; Pul, o, nucleus pulvinaris oralis; $R$, nucleus reticularis; $R e$, nucleus reuniens; $R h$, nucleus rhomboidalis; Rt, fasciculus retroflexus; $\mathrm{S}$, stria medullaris; VLc, nucleus ventralis lateralis, pars caudalis; VLm, nucleus ventralis lateralis, pars medialis; VLo, nucleus ventralis lateralis, pars oralis; VLps, nucleus ventralis lateralis, pars postrema; VPI, nucleus ventralis posterior inferior; VPLc, nucleus ventralis posterior lateralis, pars caudalis; VPLo, nucleus ventralis posterior lateralis, pars oralis; VPM, nucleus ventralis posterior medialis; $\mathrm{X}$, area $\mathrm{X}$ of Olszewski. 
clusters of cells. ${ }^{4)}$

In the area $\mathrm{X}$ of Olszewski ${ }^{8)}$ preterminal degenerating fibers were densely packed (Fig. 2, E).

In the VA a few degenerating fibers were found to make networks along its lateral border. A small number of degenerating fibers in other part of the VA and VAmc showed no definite pattern of terminations.

In addition, the LP, especially its ventromedial part, showed a moderate amount of preterminal degenerating fibers (Fig. 2, A-B). A small number of degenerating fibers found dispersedly in the $\mathrm{Li}$, SG, GMmc, Pul. $\mathrm{m}$ and Pul. 1 showed no definite pattern of terminations. Relatively many degenerating fibers in the Pul. o were also considered to be passing (Fig. 2, A). The $\mathrm{R}$ seemed to receive no cerebellar projections.

In summary, concerning the contralateral ventral thalamic nuclei preterminal degenerating fibers were densely observed in the VI (VPLo), VLc and area X, and in a less amount in the VLps after total destruction of the superior cerebellar peduncle accompanied with partial damage of the cerebellar and vestibular nuclei. According to Nauta and Mehler ${ }^{7)}$ pallidal efferent fibers terminated principally in the VA, VLo and VLm. It is interesting that the main terminal portions of cerebellar and pallidal fibers in the ventral thalamic nuclei were different in monkeys, as already suggested.2)

Some possibility of higher differentiation of the VL nuclear group in monkeys than in cats was proposed elsewhere. ${ }^{3)}$

Acknowledgement. We wish to express our gratitude to Dr. S. Honjo, Japan National Institute of Health, for his kindness concerning supply and care of the monkeys.

\section{References}

1) Fink, R. P., and Heimer, L.: Two methods for selective silver impregnation of degenerating axons and their synaptic endings in the central nervous system. Brain Research, 4, 369-374 (1967).

2) Hassler, R.: Anatomy of the Thalamus. Introduction to Stereotaxis with an Atlas of Human Brain (Eds. Schaltenbrand, G., and Bailey, P.), 1, 230-290. George Thieme, Sttutgart (1959).

3) Kusama, T., and Mabuchi, M.: Stereotaxic Atlas of the Brain of Macaca Fuscata. University of Tokyo Press, Tokyo and University Park Press, Baltimore and Manchester (1970).

4) Kusama, T.: Unpublished.

5) Mehler, W. R., Vernier, V. G., and Nauta, W. J. H.: Efferent projections from dentate and interpositus nuclei in primates. Anat. Rec., 130, 430 (1958).

6) Nauta, W. J. H., and Gygax, P. A.: Silver impregnation of degenerating axons in the central nervous system. Stain Technol., 29, 91-94 (1954). 
7) Nauta, W. J. H., and Mehler, W. R.: Projections of the lentiform nucleus in the monkey. Brain Research, 1, 3-42 (1966).

8) Olszewski, J.: The Thalamus of the Macaca Mulatta. Karger, Basel and New York (1952). 